\section{Pathologic basis of the sonographic differences between thyroid cancer and noninvasive follicular thyroid neoplasm with papillary-like nuclear features}

\author{
Grace C. H. Yang ${ }^{1}$, Karen O. Fried ${ }^{2}$ \\ 'Department of Pathology and Laboratory Medicine, Weill Medical College of Cornell \\ University, New York, NY; ${ }^{2}$ Lenox Hill Radiology, New York, NY, USA
}

Ultrasonography is pivotal in triage thyroid biopsy in the era after the identification of noninvasive follicular thyroid neoplasm with papillary-like nuclear features (NIFTP). This pictorial essay illustrates the pathologic basis of the sonographic features that distinguish NIFTP from thyroid cancers. In this study, we present the correlations of ultrasonography to $\times 1$ histopathology to assess shape and margin characteristics. Markedly hypoechoic nodules correlate to microfollicular/solid nodules, while isoechoic/hyperechoic thyroid nodules correlate to normofollicular/macrofollicular nodules. The ultrasound findings of NIFTP and minimally invasive encapsulated thyroid cancers are similar. Both are well-circumscribed, oval-to-round nodules with regular margins. Blurred or microlobulated margins indicate infiltrating tumors, while lobulated margins are characteristic of expansile tumors. Overtly invasive encapsulated tumors are characterized by oval-to-round nodules with irregular or lobulated margins. The ultrasound findings for infiltrative thyroid cancers show at least one of the following malignant features: marked hypoechoicity, taller-than-wide shape, microcalcifications, and blurred or microlobulated margins.

Keywords: Thyroid neoplasms; Thyroid ultrasound; Thyroid biopsy; Thyroid histopathology;

Thyroid fine needle aspiration cytology; Follicular variant of papillary thyroid carcinoma; Noninvasive follicular thyroid neoplasm with papillary-like nuclear features

\section{Introduction}

Before the establishment of the term noninvasive follicular thyroid neoplasm with papillarylike nuclear features (NIFTP) [1], thyroid nodules containing papillary-like nuclear features were considered cancer by definition. In the post-NIFTP era, thyroid nodules containing papillary-like nuclear features may not be considered cancer. Because of the nomenclature change, we would like to illustrate the pathologic basis for various gray-scale ultrasonography (US) features that differentiate NIFTP from thyroid cancers. Hopefully, the correlation with pathology will lead to new insights, so that that thyroid US will be even more useful in the management of thyroid nodules. We adopted

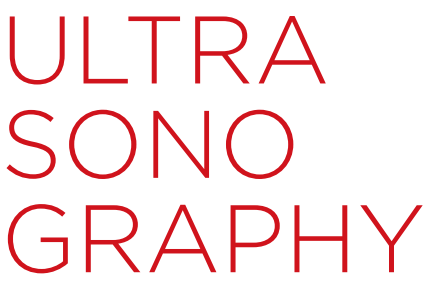

PICTORIAL ESSAY

https://doi.org/10.14366/usg. 17045 pISSN: 2288-5919 • elSSN: 2288-5943

Ultrasonography 2018;37:157-163

Received: June 24, 2017

Revised: August 5, 2017

Accepted: August 19, 2017

Correspondence to:

Grace C. H. Yang, MD, FIAC, Department of Pathology and Laboratory Medicine, Weill Medical College of Cornell University, 525 East 68 Street, Suite 766, New York, NY 10065, USA

Tel. +1-212-746-2829

Fax. +1-212-746-8359

E-mail: gry2001@med.cornell.edu

This is an Open Access article distributed under the terms of the Creative Commons Attribution NonCommercial License (http://creativecommons.org/ licenses/by-nc/3.0/) which permits unrestricted noncommercial use, distribution, and reproduction in any medium, provided the original work is properly cited.

Copyright (C) 2018 Korean Society of Ultrasound in Medicine (KSUM)

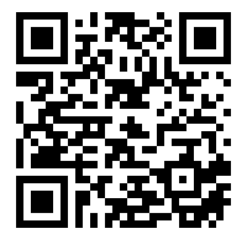

How to cite this article:

Yang GCH, Fried KO. Pathologic basis of the sonographic differences between thyroid cancer and noninvasive follicular thyroid neoplasm with papillary-like nuclear features. Ultrasonography. 2018 Apr;37(2):157-163. 
the malignant US features described in the seminal paper by Kim et al. [2], including microcalcifications, an irregular or microlobulated margin, marked hypoechogenicity, and a taller-than-wide shape (Table 1). If any of these US features was present, the nodule was classified as malignant. If a nodule had none of the features described, it was classified as benign. These criteria were validated in multiple regression analysis models in a multicenter Korean retrospective study [3]. In a recent survey of 4,696 thyroid nodules, measuring 1-2 cm [4], the guidelines proposed by Kim et al. [2] had the highest specificity, positive predictive value, and accuracy $(83.1 \%, 59.6 \%$, and $84.0 \%$, respectively) in comparison with newer
Table 1. Malignant US features of thyroid nodules as defined by Kim et al. [2]

\begin{tabular}{ll}
\hline \multicolumn{1}{c}{ Gray-scale US feature } & \multicolumn{1}{c}{ Definition } \\
\hline Microcalcifications & $\begin{array}{l}\text { Tiny, punctate hyperechoic foci-either with } \\
\text { or without acoustic shadows } \\
\text { The presence of many small lobules on the } \\
\text { surface of a nodule }\end{array}$ \\
Irregular margin & $\begin{array}{l}\text { The surface of the nodule shows irregularity } \\
\text { Decreased echogenicity compared with the } \\
\text { Taller-than-wide shape }\end{array}$ \\
& $\begin{array}{l}\text { surrounding strap muscle } \\
\text { Greater in its anteroposterior dimension } \\
\text { than its transverse dimension }\end{array}$ \\
\hline
\end{tabular}

US, ultrasonography.

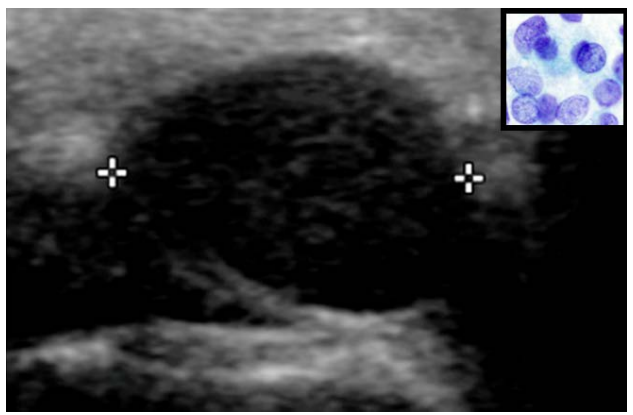

A

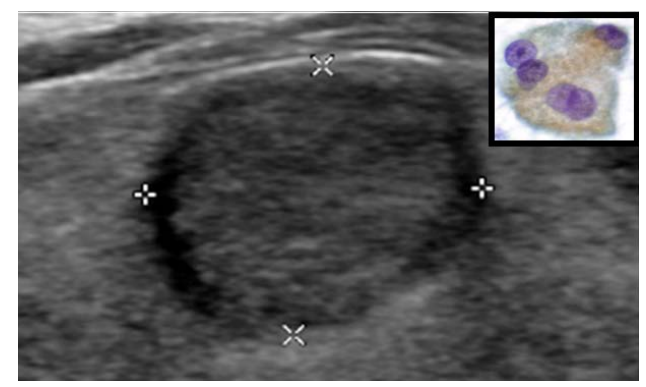

\section{D}

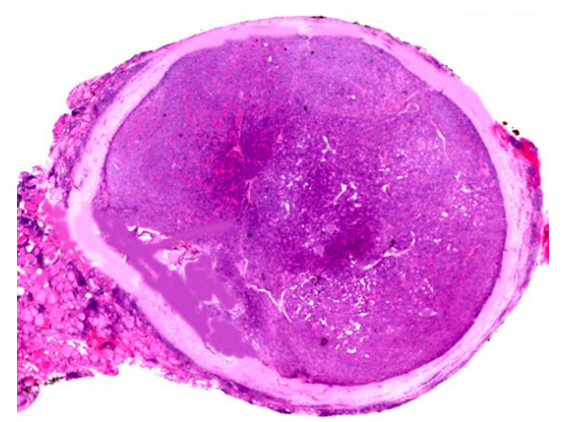

B

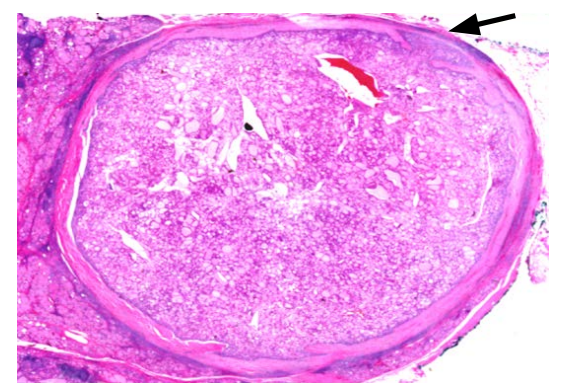

E

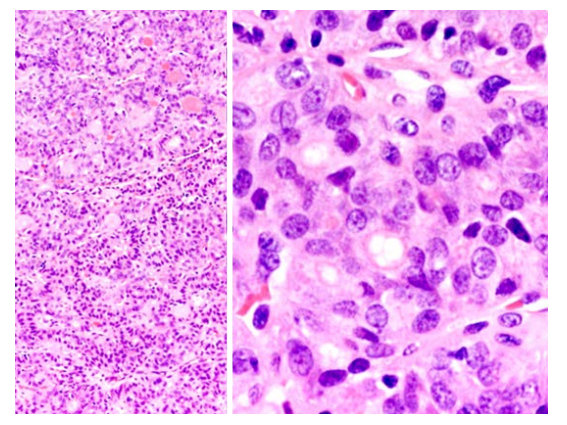

C

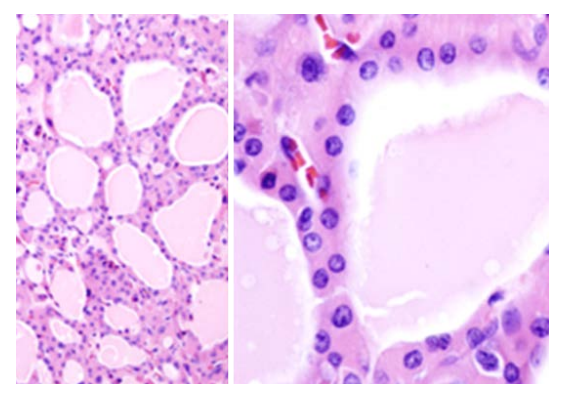

$\mathrm{F}$

Fig. 1. Microfollicular noninvasive follicular thyroid neoplasm with papillary-like nuclear features (NIFTP) (A-C) versus encapsulated macrofollicular Hürthle cell carcinoma with microscopic capsular invasion (D-F).

A-C. The top case is from a 49-year-old woman with a $1.5-\mathrm{cm}$ right upper thyroid nodule. A. Ultrasonography shows a well-circumscribed, oval-to-round, markedly hypoechoic nodule (inset, Papanicolaou, $\times 600$; microfollicles with mixed normal and papillary-like oval and clear nuclei aspirated). Fine needle aspiration (FNA): cannot exclude follicular variant of papillary thyroid carcinoma (Bethesda V/VI). B. Hemithyroidectomy shows an oval-round blue nodule with a thick capsule $(H \& E, \times 1)$. The thick capsule was invisible on ultrasonography, because the echogenicity of the capsule and the tumor were both markedly hypoechoic. Notice that the black outlines of the cells in the insets disappear in the black background. C. The blue nodule was composed of microfollicles (left) and the cells had grooved, oval papillarylike nuclei with mild chromatin clearing (right). No evidence for invasion on thorough sampling and no increased mitotic figures or necrosis were present, so the final diagnosis was NIFTP (left, H\&E, $\times 100$; right, H\&E, $\times 400$ ). D-F. The bottom case is from a 62 -year-old woman with a 1.4-cm right middle thyroid nodule. D. Ultrasonography shows a well-circumscribed, oval-to-round, hypoechoic nodule outlined by a markedly hypoechoic rim (inset, Papanicolaou, $\times 600$; Hürthle cells with abundant granular cytoplasm containing non-papillary nuclei [round, granular chromatin, prominent nucleoli] along with abundant thin colloid aspirated). FNA: Hürthle cell nodule (Bethesda III/VI). E. Histopathology shows a pink oval-to-round nodule with multifocal microscopic capsular invasion (arrow points to one) undetectable by ultrasound $(H \& E, \times 1)$. F. Colloid-filled macrofollicular nodule (left) composed of Hürthle cells with abundant granular cytoplasm with nonpapillary nuclei (round with prominent nucleoli) (right); the final diagnosis was minimally invasive Hürthle cell carcinoma (left, $H \& E, \times 100$; right, $H \& E, \times 400)$. 
international guidelines for thyroid nodules [5-7].

\section{Ultrasound-Guided Fine Needle Aspiration and Data Collection}

As described previously [8], thyroid nodules were sampled by a radiologist with a $27 \mathrm{G}$ needle, guided by a sonographer using a 7-15 MHz linear array "hockey stick" probe on the Phillips IU22 ultrasound system (Philips Healthcare, Andover, MA, USA) and immediately assessed by a cytopathologist. The ultrasound findings were entered into a FileMaker Pro database (FileMaker, Inc., Santa Clara, CA, USA) on a laptop by the cytopathologist. Following surgery, the pre-fine needle aspiration (FNA) ultrasound images were obtained from radiology and correlated with the histopathology of the index nodule for shape, echogenicity, and margin characteristics. Hematoxylin and eosin (H\&E) stained sections were placed on a white background for gross inspection. The H\&E section best correlating to the pre-FNA gray-scale US image was selected and then scanned at 800 dpi on a UMAX Powerlook III flatbed scanner (Fremont, CA, USA). The pre-FNA gray-scale and color Doppler ultrasound images along with histology images taken at $\times 1, \times 20, \times 40, \times 100$, and $\times 400$ of each case were then filed under the histopathology classification.

Over a 21-year-period, 698 cases had surgical follow-up. Preoperative US and the histopathology of index nodules were correlated. The data were collected from 557 women and 141 men, with a mean age of 48 years (range, 16 to 87 years). There were 273 benign neoplastic nodules, including 226 follicular adenoma/

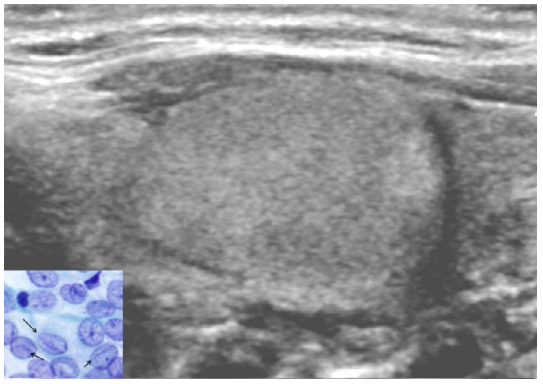

A

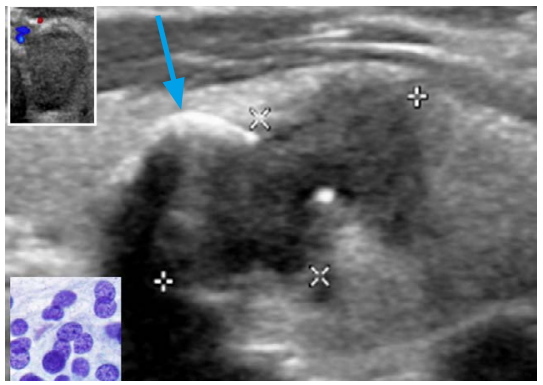

D

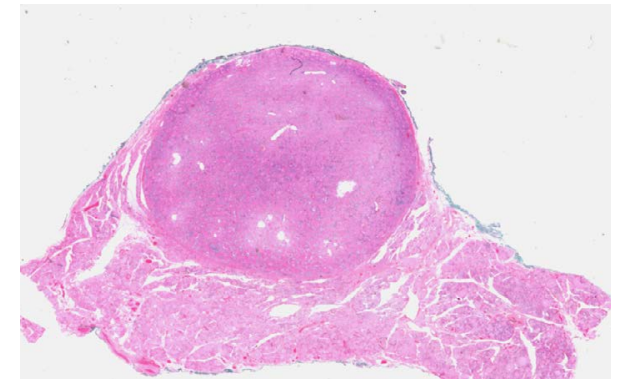

B

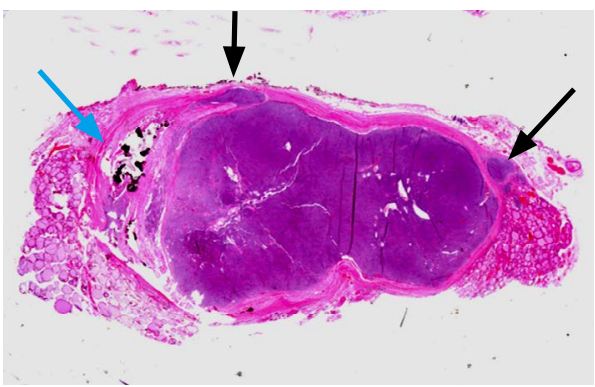

E

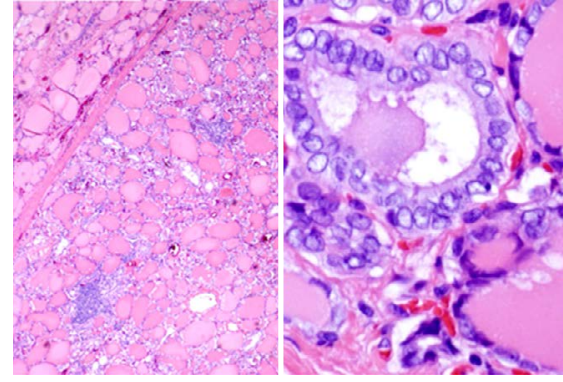

C

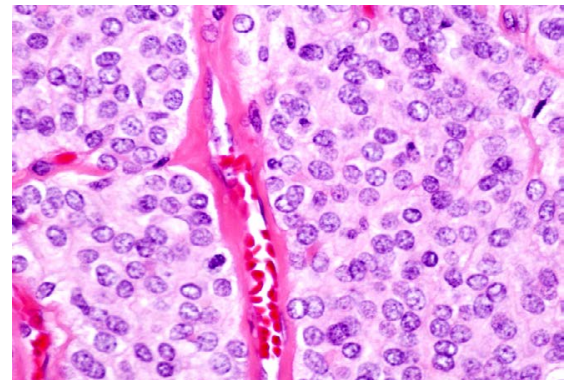

$\mathrm{F}$

Fig. 2. Normofollicular noninvasive follicular thyroid neoplasm with papillary-like nuclear features $(A-C)$ versus poorly differentiated thyroid carcinoma (D-F).

A-C. The top case is from a 68 -year-old woman with a $2.7-\mathrm{cm}$ right lobe thyroid nodule. A. Ultrasonography shows an oval, isoechoic, homogeneous nodule with regular margins and hypoechoic rim (inset, Papanicolaou, $\times 600$; oval clear nuclei with grooves [arrows] aspirated). Fine needle aspiration (FNA): suspicious for follicular variant of papillary thyroid carcinoma (Bethesda V/VI). B. Hemithyroidectomy shows an encapsulated, oval, pink nodule and the capsule correlated to the hypoechoic rim on ultrasonography (H\&E, $\times 1)$. C. The nodule was pink because it was composed of normal-sized follicles filled with pink colloid. Notice the thin capsule at left upper corner. The papillarylike nuclei were oval, grooved, and clear (left, H\&E, $\times 100$; right, H\&E, $\times 400$ ). D-F. The bottom case is from a 63-year-old woman with a 1.7$\mathrm{cm}$ left lobe thyroid nodule. D. Ultrasonography shows a markedly hypoechoic nodule with lobulated margins and coarse rim calcification (blue arrow) (top inset, the markedly hypoechoic nodule had a taller-than-wide shape on transverse view; bottom inset, Papanicolaou, $\times 600$; aspirated cells with small round nuclei). FNA: suspicious for thyroid carcinoma (Bethesda V/VI). E. Histopathology shows an encapsulated blue nodule with expansile growth forming lobulated margins. The blue arrow points to coarse calcifications along a thick capsule. The black arrows point to capsular invasions $(H \& E, \times 1)$. F. The nodule was blue due to the high nuclear density from the solid tumor nests. Similar to on FNA, the tumor has small, round nuclei $(H \& E, \times 400)$. 


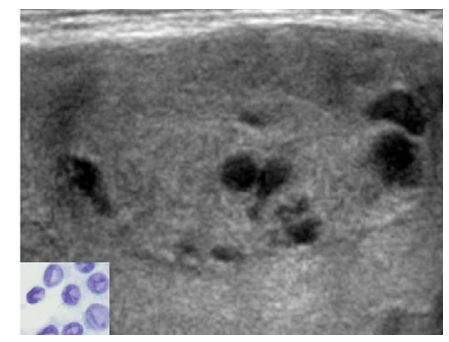

A

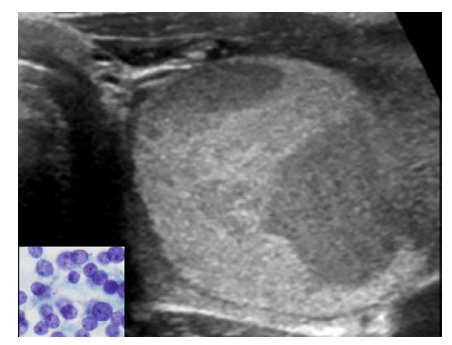

$\mathrm{E}$

Fig. 3. Macrofollicular noninvasive follicular thyroid neoplasm with papillary-like nuclear features with cysts (A-D) versus minimally invasive follicular thyroid carcinoma with sharp demarcated microfollicular and macrofollicular components $(\mathrm{E}-\mathrm{H})$.

A-D. The top case was from a 37-year-old woman with a 3-cm right lobe thyroid nodule. A. An oval, isoechoic nodule was outlined by a thin hypoechoic rim on ultrasonography. The composition of the nodule was $\sim 85 \%$ solid and $\sim 15 \%$ cysts. Color Doppler ultrasonography shows mainly peripheral vascularity. Abundant thin colloid and sheets of epithelium with oval grooved papillary-like nuclei (inset, Papanicolaou, $\times 600$ ) were aspirated. Fine needle aspiration (FNA): suspicious for macro-follicular variant of papillary thyroid carcinoma (Bethesda V/VI). B. Hemithyroidectomy shows a circumscribed solid cystic pink nodule with similar intensity of pinkness as the surrounding thyroid, correlating to the isoechoic nodule with a thin rim on ultrasonography $(H \& E, \times 1)$. C. The macrofollicular nodule had a thin capsule (arrow) $(H \& E, \times 20)$. D. The papillary-like nuclei along the colloid lake were elongated, grooved, and clear (H\&E, $\times 400)$. E-H. The bottom case was from a 38-year-old woman with a 4.5-cm hypervascular nodule replacing the left lobe. E. A well-circumscribed hyperechoic nodule with hypoechoic rim contained sharply demarcated hypoechoic regions (inset, Papanicolaou, $\times 600$; microfollicles with small, round, and dark nuclei aspirated from the hypoechoic region). FNA: follicular neoplasm (Bethesda IV/VI). F. Hemithyroidectomy shows a blue-pink two-toned nodule with a thick capsule $(H \& E, \times 1)$. G. Sharply demarcated microfollicular region was present $(H \& E, \times 20)$ (Inset, $\times 20$; a single focus of capsular invasion was found in 12 sections on microscopic examination). H. Both the macrofollicular region (left) and microfollicular region (right) had small, round, and granular non-papillary nuclei $(H \& E, \times 400)$.

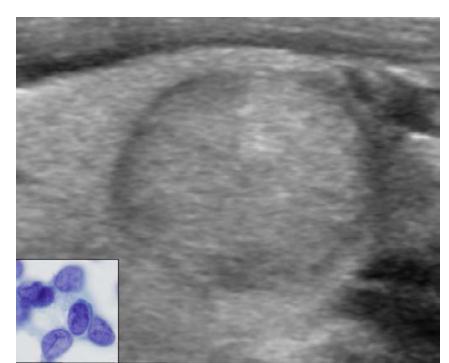

A

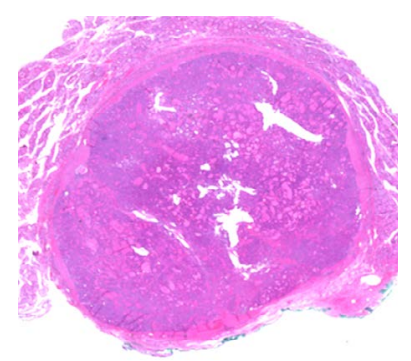

B

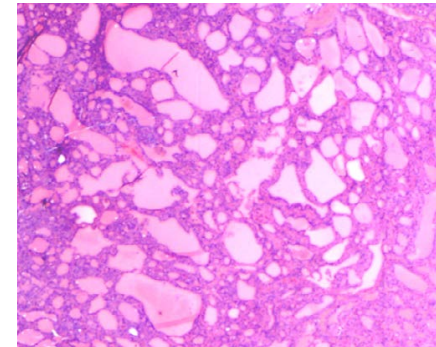

C

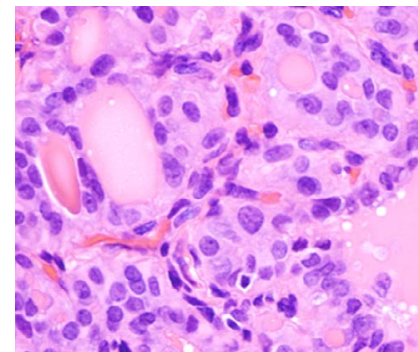

D

Fig. 4. Normofollicular noninvasive follicular thyroid neoplasm with papillary-like nuclear features (A-D) versus microfollicular follicular thyroid carcinoma with overt capsular invasions $(\mathrm{E}-\mathrm{H})$.

A-D. The case is from a 56-year-old man with a 1.6-cm right lower thyroid nodule. A. A round, isoechoic nodule is outlined by a hypoechoic rim on ultrasonography. Enlarged, oval, grooved, papillary-like nuclei (inset, Papanicolaou, $\times 600$ ), along with $90 \%$ normal nuclei were present in the aspirate. Fine needle aspiration (FNA): cannot exclude follicular variant of papillary thyroid carcinoma (Bethesda VNII). B. Hemithyroidectomy shows an encapsulated blue nodule with minute pink dots and lines. Neither capsular nor vascular invasions were found on thorough sampling and examination $(H \& E, \times 1)$. C. The nodule was composed of mixed large and small follicles (H\&E, $\times 40)$. D. The papillary-like nuclei were enlarged, oval, and grooved. The chromatin was dark, but powdery $(\mathrm{H \& E}, \times 400)$. 


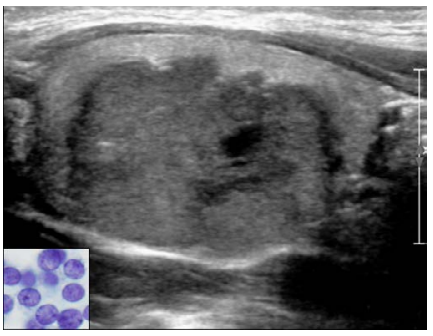

$\mathrm{E}$

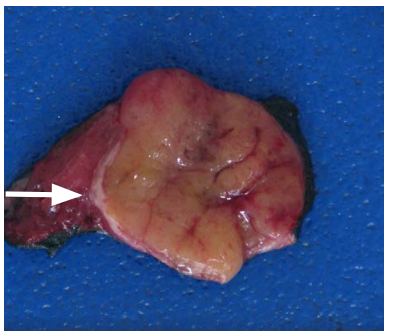

$\mathrm{F}$

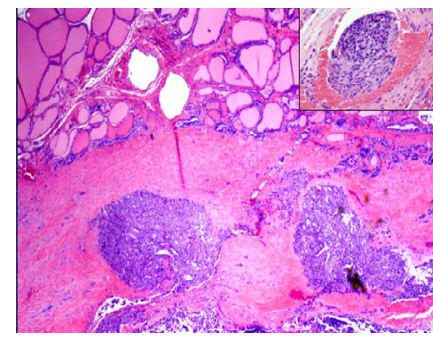

G

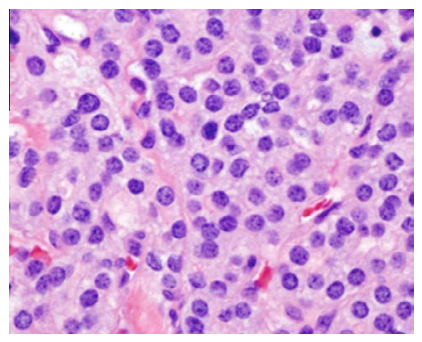

$\mathrm{H}$

E-H. The case bottom case is from a 27-year-old woman with a 3.3-cm right mid-lower nodule. E. An oval, homogeneous hypoechoic nodule with irregular borders is outlined by a hypoechoic rim on ultrasonography (inset, Papanicolaou, $\times 600$; microfollicles with small, round, and granular non-papillary nuclei were aspirated). FNA: suspicious for thyroid carcinoma (Bethesda V/VI). F. The cut surface shows a bulging fleshy tan encapsulated (arrow) tumor with irregular margins. G. Thick capsule with overt capsular invasions and four microscopic angioinvasions (inset, $\times 100)$ were present $(H \& E, \times 20)$. H. Microfollicular tumor with small, round, and granular non-papillary nuclei was present $(H \& E$, $\times 400)$.

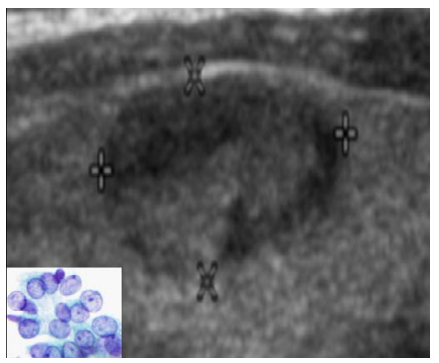

A

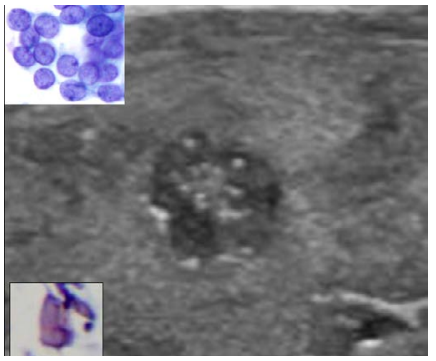

$\mathrm{E}$

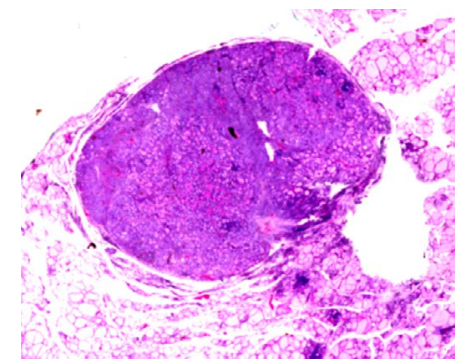

B

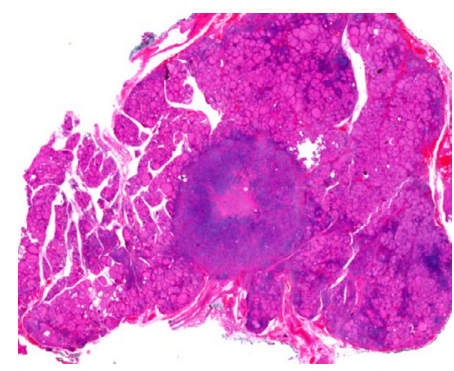

$\mathrm{F}$

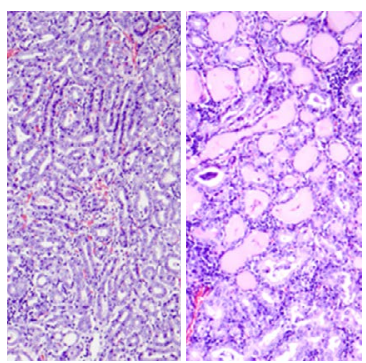

C

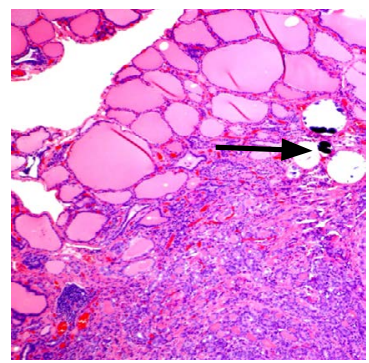

G

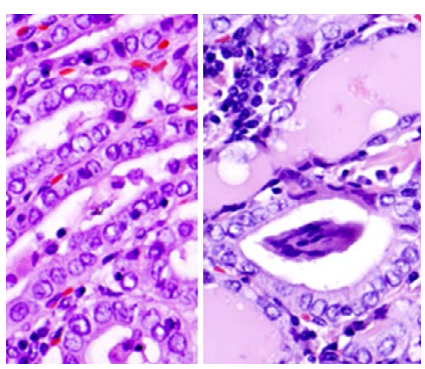

D

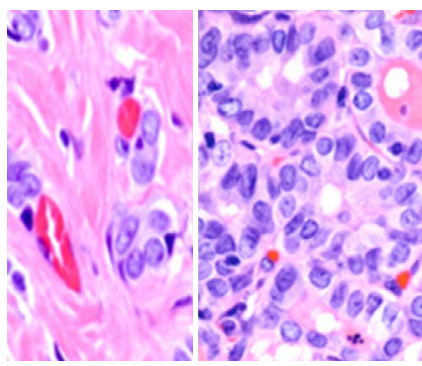

$\mathrm{H}$

Fig. 5. Mixed microfollicular versus normofollicular noninvasive follicular thyroid neoplasm with papillary-like nuclear features (A-D) and infiltrative follicular variant of papillary thyroid carcinoma (FVPTC) with irregular borders and microcalcifications (E-H).

A-D. The top case is from a 37-year-old woman with a $1.5-\mathrm{cm}$ right mid-lower thyroid nodule. A. Oval, solid, hypoechoic (top third) and isoechoic (bottom two-thirds) nodule, outlined by a hypoechoic rim that was visible only along the isoechoic region was found on ultrasonography (inset, Papanicolaou, $\times 600$; microfollicles with enlarged, oval, papillary-like nuclei aspirated). Fine needle aspiration (FNA): suspicious for FVPTC (Bethesda V/VI). B. Hemithyroidectomy shows a thinly encapsulated blue nodule with minute pink dots and lines. No invasion was found on microscopic examination $(H \& E, \times 1)$. C. The nodule had both microfollicular (left) and normofollicular areas (right) (H\&E, $\times 100$ ). D. The papillary-like nuclei with clear oval nuclei were present in both the microfollicular (left) and normofollicular (right) regions (H\&E, $\times 400$ ). E-H. The bottom case is from a 25 -year-old woman with a $0.8-\mathrm{cm}$ right middle thyroid nodule. E. A markedly hypoechoic nodule with microcalcifications, echogenic center, and microlobulated margins was found on ultrasonography. Specks of calcifications and microfollicles with enlarged, oval, papillary-like nuclei with powdery chromatin were aspirated (insets, Papanicolaou, $\times 600$ ). FNA: FVPTC (Bethesda VI/VI). F. Histopathology shows a blue nodule with a pink center and fussy margins $(H \& E, \times 1)$. G. Tumor microfollicles infiltrated into surrounding pink colloid-containing normal thyroid. The arrow points to the residual calcifications surrounded by 3 holes, from microcalcifications that popped out during sectioning of the paraffin block (H\&E, $\times 40)$. H. The pink center of the nodule is due to a fibrotic reaction to the infiltrating microfollicles (left); the blue region of the nodule had high nuclear density due to back-to-back microfollicles with enlarged, oval, papillarylike nuclei with powdery chromatin (right) (H\&E, ×400). 
adenomatoid nodules, 42 Hürthle cell adenoma/adenomatous nodules, and five hyalinizing trabecular adenomas. Among the 425 malignant thyroid nodules, two were anaplastic carcinomas, five were poorly differentiated thyroid carcinomas; six were follicular thyroid carcinomas; 12 were Hürthle cell carcinomas; nine were medullary carcinomas; and 391 were papillary thyroid carcinomas (PTC). Cases of the follicular variant of PTC (FVPTC) were reclassified into NIFTP, the invasive encapsulated follicular variant of PTC, and the infiltrative FVPTC in a recent review [9]. The corresponding color Doppler data were reported in a recent study [8]. The most educational cases illustrating various gray-scale US features were chosen for this pictorial essay.

\section{Results}

The NIFTP cases were oval to round nodules with regular margins. The capsule appeared as a hypoechoic rim, except when the nodules were markedly hypoechoic, as illustrated in Fig. 1A and B. The echogenicity of NIFTP cases was variable, ranging from markedly hypoechoic (Fig. 1) to hypoechoic (Fig. 2), isoechoic (Figs. 3, 4), and mixed hypoechoic and isoechoic (Fig. 5). One NIFTP had cystic changes (Fig. 3). The NIFTP case without a hypoechoic rim, illustrated in Fig. 1, had a thick capsule, but was invisible on the gray-scale US due to the equally marked hypoechogenicity of the nodule composed of back-to-back microfollicles without colloid.

Table 2. Pathologic basis of ultrasound differences between NIFTP and thyroid cancers

\begin{tabular}{|c|c|c|c|c|}
\hline & Gray-scale ultrasound & $\times 1$ Histopathology & Tumor type & \\
\hline \multirow[t]{4}{*}{ Shape } & Oval to round & Oval to round & NIFTP cases & Figs. $1-5$ \\
\hline & & & Minimally invasive $\mathrm{HCa}$ & Fig. 1 \\
\hline & & & Minimally invasive FTC & Fig. 3 \\
\hline & Taller-than-wide & Vertical growth & PDTC & Fig. 2 (top inset) \\
\hline \multirow[t]{6}{*}{ Margin } & Well-defined, smooth & No invasion or microscopic invasions & NIFTP cases & Figs. $1-5$ \\
\hline & & & Minimally invasive $\mathrm{HCa}$ & Fig. 1 \\
\hline & & & Minimally invasive FTC & Fig. 3 \\
\hline & Lobulated & $\begin{array}{l}\text { Expansile growth, group of tumor cells } \\
\text { "pushing" into surrounding follicles }\end{array}$ & PDTC & Fig. 2 \\
\hline & Microlobulated, blurred & $\begin{array}{l}\text { Tumor cells insidiously infiltrating into } \\
\text { surrounding follicles }\end{array}$ & IFVPTC & Fig. 5 \\
\hline & Irregular & Irregular & Overtly invasive FTC & Fig. 4 \\
\hline \multirow[t]{9}{*}{ Echogenicity } & Marked hypoechoic & $\begin{array}{l}\text { Tightly packed microfollicles or solid nests of } \\
\text { tumor cells }\end{array}$ & NIFTP & Fig. 1 \\
\hline & & & PDTC & Fig. 2 \\
\hline & Hypoechoic & Small-to-medium follicles & NIFTP cases & Figs. 4,5 \\
\hline & & & Minimally invasive FTC & Fig. 3 \\
\hline & & & Overtly invasive FTC & Fig. 4 \\
\hline & & & IFVPTC & Fig. 5 \\
\hline & Isoechoic to hyperechoic & Normal-to-macrofollicles & NIFTP cases & Figs. 2, 3 \\
\hline & & & Minimally invasive $\mathrm{HCa}$ & Fig. 1 \\
\hline & & & Minimally invasive FTC & Fig. 3 \\
\hline \multirow[t]{5}{*}{ Hypoechoic rim (halo) } & Visible & $\begin{array}{l}\text { Capsule around normal-sized follicles or } \\
\text { macrofollicles }\end{array}$ & NIFTP cases & Figs. 2-5 \\
\hline & & & Minimally invasive $\mathrm{HCa}$ & Fig. 1 \\
\hline & & & Minimally invasive FTC & Fig. 3 \\
\hline & & & Overtly invasive FTC & Fig. 4 \\
\hline & Invisible & Tightly packed microfollicles & NIFTP & Fig. 1 \\
\hline \multirow[t]{2}{*}{ Calcifications } & Micro & Non-psammomatous small calcifications & IFVPTC & Fig. 5 \\
\hline & $\operatorname{Rim}$ & Coarse calcifications & PDTC & Fig. 2 \\
\hline Solid-cystic composition & Isoechoic nodule with cystic areas & Macrofollicular nodule with cystic areas & NIFTP & Fig. 3 \\
\hline
\end{tabular}

NIFTP, noninvasive follicular thyroid neoplasm with papillary-like nuclear features; HCa, Hürthle cell carcinoma (=FTC, oncocytic variant); FTC, follicular thyroid carcinoma; PDTC, poorly differentiated thyroid carcinoma; IFVPTC, infiltrative follicular variant of papillary thyroid carcinoma. 
Five NIFTP cases are contrasted with four cases of encapsulated thyroid cancer and one case of infiltrative thyroid cancer. Fig. 1 shows a minimally invasive encapsulated macrofollicular Hürthle cell carcinoma with a benign US appearance. Fig. 2 illustrates a poorly differentiated thyroid carcinoma that presented on US as a dumbbell-shaped, markedly hypoechoic nodule with lobulated margins and coarse rim calcifications. On a transverse view, the nodule appeared taller than wide. Fig. 3 illustrates a minimally invasive follicular thyroid carcinoma with a well-defined margin, but showing sharply demarcated hypoechoic-hyperechoic regions. Fig. 4 illustrates an overtly invasive follicular thyroid carcinoma as an oval, hypoechoic nodule with irregular margins. Fig. 5 shows an infiltrative FVPTC with microlobulated margins and microcalcifications. The FNA cytology reports with Bethesda classifications for each illustrated case are in the figure legends.

The correlation of histopathology with various gray-scale thyroid US features is shown in Table 2.

\section{Summary}

Scanned H\&E sections of thyroid nodules were correlated with thyroid ultrasound. On histology, the thyroid gland is composed mainly of follicles. Simple cuboidal epithelium made of follicular cells surrounds colloid. The luminal colloid is stained pink by eosin, and the nucleus of follicular cells is stained blue by hematoxylin. The size of the follicles is inversely proportional to the echogenicity. Thyroid nodules containing small follicles (i.e., microfollicular nodules) have many more nuclei per unit area (i.e., high nuclear density), and thus appear blue on H\&E sections and markedly hypoechoic on gray-scale ultrasound. In contrast, thyroid nodules containing large follicles (i.e., macrofollicular nodules) have low nuclear density, and thus appear pink on H\&E sections and isoechoic or hyperechoic on US. The capsule is composed of fibrotic tissue, which also blocks sound waves; for this reason, it appears as a hypoechoic rim around mildly hypoechoic, isoechoic, or hyperechoic nodules, but is invisible around markedly hypoechoic nodules. The margin characteristics of thyroid nodules in H\&E sections also correlate with the ultrasound findings. US can detect overt, grossly visible invasions, but identifying minimal invasions or angioinvasion requires the microscopic examination of a thoroughly sampled capsule on histopathology. Microcalcifications on US correlate to fine calcifications on both cytology and histology, but not necessarily psammoma bodies with concentric rings of calcification associated with papillae.
ORCID: Grace C. H. Yang: http://orcid.org/0000-0002-4797-1272

\section{Conflict of Interest}

No potential conflict of interest relevant to this article was reported.

\section{References}

1. Nikiforov YE, Seethala RR, Tallini G, Baloch ZW, Basolo F, Thompson $L D$, et al. Nomenclature revision for encapsulated follicular variant of papillary thyroid carcinoma: a paradigm shift to reduce overtreatment of indolent tumors. JAMA Oncol 2016;2:1023-1029.

2. Kim EK, Park CS, Chung WY, Oh KK, Kim DI, Lee JT, et al. New sonographic criteria for recommending fine-needle aspiration biopsy of nonpalpable solid nodules of the thyroid. AJR Am J Roentgenol 2002;178:687-691.

3. Kwak JY, Jung I, Baek JH, Baek SM, Choi N, Choi YJ, et al. Image reporting and characterization system for ultrasound features of thyroid nodules: multicentric Korean retrospective study. Korean J Radiol 2013;14:110-117.

4. Yoon JH, Han K, Kim EK, Moon HJ, Kwak JY. Diagnosis and management of small thyroid nodules: a comparative study with six guidelines for thyroid nodules. Radiology 2017;283:560-569.

5. Kwak JY, Han KH, Yoon JH, Moon HJ, Son EJ, Park SH, et al. Thyroid imaging reporting and data system for US features of nodules: a step in establishing better stratification of cancer risk. Radiology 2011;260:892-899.

6. Russ G, Royer B, Bigorgne C, Rouxel A, Bienvenu-Perrard M, Leenhardt L. Prospective evaluation of thyroid imaging reporting and data system on 4550 nodules with and without elastography. Eur J Endocrinol 2013;168:649-655.

7. Haugen BR, Alexander EK, Bible KC, Doherty GM, Mandel SJ, Nikiforov YE, et al. 2015 American Thyroid Association management guidelines for adult patients with thyroid nodules and differentiated thyroid cancer: the American Thyroid Association Guidelines Task Force on Thyroid Nodules and Differentiated Thyroid Cancer. Thyroid 2016;26:1-133.

8. Yang GC, Fried KO. Most thyroid cancers detected by sonography lack intranodular vascularity on color Doppler imaging: review of the literature and sonographic-pathologic correlations for 698 thyroid neoplasms. J Ultrasound Med 2017;36:89-94.

9. Yang GC, Fried KO, Scognamiglio T. Sonographic and cytologic differences of NIFTP from infiltrative or invasive encapsulated follicular variant of papillary thyroid carcinoma: A Review of 179 Cases. Diagn Cytopathol 2017;45:533-541. 